\title{
Lack of atropine in Syria hampers treatment after gas attacks
}

This News article incorrectly states that the charity Médecins Sans Frontières does not have any staff in Syria (BMJ 2013;347:f5413, doi:10.1136/bmj.f5413). In fact, MSF has since communicated to us that it directly operates six hospitals and four health centres in the north of Syria and, in areas where
MSF cannot send its own teams because of insecurity or lack of access, the charity supports a network of Syrian doctors.

Cite this as: BMJ 2013;347:f5530

๑ BMJ Publishing Group Ltd 2013 